\title{
A NOTE ON THE ZEROS OF THE SECTIONS OF A PARTIAL FRACTION
}

\author{
MORRIS MARDEN
}

1. Introduction. If $f(z)$ is a rational function with a total of three distinct zeros and poles, the zeros of its logarithmic derivative may be located as points in the complex plane by aid of the following theorem.

THEOREM 1. The zeros of the partial fraction

$$
F(z)=\frac{m_{1}}{z-z_{1}}+\frac{m_{2}}{z-z_{2}}+\frac{m_{3}}{z-z_{3}}, \quad m_{1} m_{2} m_{3} \neq 0,
$$

where $z_{1}, z_{2}$ and $z_{3}$ are three distinct, noncollinear points, lie at the foci of the conic which touches the line segments $\left(z_{2}, z_{3}\right),\left(z_{3}, z_{1}\right)$ and $\left(z_{1}, z_{2}\right)$ in the points $\zeta_{1}, \zeta_{2}$ and $\zeta_{3}$ that divide these segments in the ratio $m_{2}: m_{3}$, $m_{3}: m_{1}$, and $m_{1}: m_{2}$ respectively. If $n=m_{1}+m_{2}+m_{3} \neq 0$, this conic is an ellipse or hyperbola according as $n m_{1} m_{2} m_{3}>0$ or $<0$. If $n=0$, the conic is a parabola whose axis is parallel to the line joining the origin to the point $\nu=m_{1} z_{1}+m_{2} z_{2}+m_{3} z_{3}$.

In the special case $m_{1}=m_{2}=m_{3}=1$, this theorem was proved geometrically by Bôcher and Grace. ${ }^{1}$ In the general case it was first deduced by Linfield as a corollary to the following theorem which in turn was established by the use of line coordinates and polar forms. ${ }^{2}$

THEOREM 2. The zeros of the partial fraction $F(z)=\sum_{j=1}^{p} m_{j} /\left(z-z_{j}\right)$ lie at the foci of the curve $C\left(z_{1}, z_{2}, \cdots, z_{p} ; m_{1}, m_{2}, \cdots, m_{p}\right)$ of class $p-1$ which touches each of the $p(p-1) / 2$ line-segments $\left(z_{j}, z_{k}\right)$ in a point dividing it in the ratio $m_{j}: m_{k}$.

In view, however, of the elementary character of Theorem 1, it

Presented to the Society, April 28, 1945; received by the editors April 23, 1945.

${ }^{1}$ M. Bôcher, Ann. of Math. vol. 7 (1892) pp. 70-76; J. H. Grace, Proc. Cambridge Philos. Soc. vol. 11 (1901) pp. 352-357.

${ }^{2}$ For the case that all $m_{j}>0$, see Siebeck, J. Reine Angew. Math. vol. 64 (1864) p. 175; M. Van den Berg, Niew Archief voor Wiskunde vol. 9 (1882) pp. 1-14, 60, vol. 11 (1884) pp. 153-186, vol. 15 (1899) pp. 100-164; J. Juhel-Renjoy, C. R. Acad. Sci. Paris vol. 142 (1906); P. J. Heawood, Quart. J. Math. vol. 38 (1907). pp. 84-107; and M. Fujiwara, Tôhoku Math. J. vol. 9 (1916) pp. 102-108. It is to be observed that, although priority for the theorem when all $m_{j}>0$ is usually accorded to Van den Berg, it should rightfully be given to Siebeck. For arbitrary integral $m_{j}$, see B. Z. Linfield, Bull. Amer. Math. Soc. vol. 27 (1920) pp. 17-21 and Trans. Amer. Math. Soc. vol. 25 (1923) pp. 239-258. 
seems desirable to furnish for it an elementary proof based upon some familiar property of the conics. In the next paragraph we shall construct such a proof, based upon the optical properties of the conics and upon some apparently new propositions concerning the relative positions of the zeros of the partial fraction $F(z)$, and those of its two sections, $F_{1}(z)=\sum_{1}^{\boldsymbol{k}} m_{j}\left(z-z_{j}\right)^{-1}$ and $F_{2}(z)=\sum_{k+1}^{p} m_{j}\left(z-z_{j}\right)^{-1}$. These propositions, which will be derived in $\$ \S 2$ and 3 by the aid of very simple analysis, will also be used in $\$ 4$ together with Theorem 2 to obtain some relations among the foci of the curves $C\left(z_{1}, z_{2}, \cdots, z_{k}\right.$; $\left.m_{1}, m_{2}, \cdots, m_{k}\right), C\left(z_{k+1}, \cdots, z_{p} ; m_{k+1}, \cdots, m_{p}\right)$ and $C\left(z_{1}, z_{2}, \cdots, z_{p}\right.$; $\left.m_{1}, m_{2}, \cdots, m_{p}\right)$. Finally, in $\$ 5$, applications of the method will be indicated for nonlinear, partial fractions.

2. Proof of Theorem 1. Assuming $n \neq 0$ and $n_{3}=m_{1}+m_{2} \neq 0$, let us write

$$
F(z)=\frac{n\left(z-Z_{1}\right)\left(z-Z_{2}\right)}{\left(z-z_{1}\right)\left(z-z_{2}\right)\left(z-z_{3}\right)} .
$$

Since by definition

$$
n_{3} \zeta_{3}=m_{2} z_{1}+m_{1} z_{2},
$$

it follows that $\zeta_{3}$ is the zero of the partial fraction $F_{1}(z)=m_{1} /\left(z-z_{1}\right)$ $+m_{2} /\left(z-z_{2}\right)$. Hence,

$$
\frac{\zeta_{3}-z_{3}}{n} F\left(\zeta_{3}\right)=\frac{m_{3}}{n}=\frac{\left(Z_{1}-\zeta_{3}\right)\left(Z_{2}-\zeta_{3}\right)}{\left(z_{1}-\zeta_{3}\right)\left(z_{2}-\zeta_{3}\right)},
$$

$$
\arg \left(Z_{1}-\zeta_{3}\right) /\left(z_{1}-\zeta_{3}\right) \equiv \arg \left(z_{2}-\zeta_{3}\right) /\left(Z_{2}-\zeta_{3}\right)
$$

$(\bmod 2 \pi)$ if $n m_{3}>0$,

$$
\arg \left(Z_{1}-\zeta_{3}\right) /\left(z_{1}-\zeta_{3}\right) \equiv \arg \left(\zeta_{3}-z_{2}\right) /\left(Z_{2}-\zeta_{3}\right)
$$

$(\bmod 2 \pi)$ if $n m_{3}<0$.

By clearing equation (2.1) of fractions and equating the coefficients of $z$ on both sides of the resulting equation, we learn that

$$
\left(Z_{1}+Z_{2}\right) / 2=\left(n_{1} z_{1}+n_{2} z_{2}+n_{3} z_{3}\right) / n
$$

where $n_{1}=m_{2}+m_{3}, n_{2}=m_{3}+m_{1}$ and $n_{3}=m_{1}+m_{2}$. Since by hypothesis $n_{3} \neq 0$, the point $\left(Z_{1}+Z_{2}\right) / 2$ cannot lie on the segment $\left(z_{1}, z_{2}\right)$ and hence not both $Z_{1}$ and $Z_{2}$ may lie on this segment.

If $\zeta_{3}$ is an interior division point of the line segment $\left(z_{1}, z_{2}\right)$ and thus $m_{1} m_{2}>0$, we learn from equations (2.3) and (2.4) that the line $\left(z_{1}, z_{2}\right)$ makes equal angles with lines $\left(Z_{1}, \zeta_{3}\right)$ and $\left(Z_{2}, \zeta_{3}\right)$ and does or does not separate the points $Z_{1}$ and $Z_{2}$ according as $n m_{3}<0$ or $>0$. 
Likewise, if $\zeta_{3}$ is an exterior division point of the line segment $\left(z_{1}, z_{2}\right)$ and thus $m_{1} m_{2}<0$, we learn from (2.3) and (2.4) that the line $\left(z_{1}, z_{2}\right)$ makes equal angles with the lines $\left(Z_{1}, \zeta_{3}\right)$ and $\left(Z_{2}, \zeta_{3}\right)$ and does or does not separate the points $Z_{1}$ and $Z_{2}$ according as $n m_{3}>0$ or $<0$. Hence, using the optical properties of the conics, we infer that at point $\zeta_{3}$ the line $\left(z_{1}, z_{2}\right)$ is tangent to a conic with foci at points $Z_{1}$ and $Z_{2}$ and that this conic is an ellipse or hyperbola according as $n m_{1} m_{2} m_{3}>0$ or $<0$.

Now, by merely permuting the subscripts 1,2 , and 3 , we may complete the proof of the part of the theorem involving the ellipse and hyperbola.

To prove the part involving the parabola, let us, since $n=0$, write instead of (2.1) the expression

$$
F(z)=\frac{\nu\left(z-Z_{1}\right)}{\left(z-z_{1}\right)\left(z-z_{2}\right)\left(z-z_{3}\right)} .
$$

The quantity $\nu=m_{1} z_{1}+m_{2} z_{2}+m_{3} z_{3} \neq 0$ because, by hypothesis, the points $z_{1}, z_{2}$ and $z_{3}$ are noncollinear. Since now $\nu$ replaces the factor $\left(z-Z_{2}\right)$, a repetition of the above details would prove at once that line $\left(z_{1}, z_{2}\right)$ is tangent at point $\zeta_{3}$ to the parabola described in Theorem 1 .

Remark. It is obvious from the proof that Theorem 1 holds not only, as usually asserted, when the $m_{i}$ are integers but also when they are arbitrary real numbers. Using this fact, we may extend our theorem to the case that, for example, $n_{3}=m_{1}+m_{2}=0$ but $n \neq 0$. For, taking $n_{3}$ very small, we find that $n m_{3}>0, m_{1} m_{2}<0$, and, hence, $n m_{1} m_{2} m_{3}<0$. The corresponding conic is therefore a hyperbola which is tangent at $\zeta_{3}$ to the line $\left(z_{1}, z_{2}\right)$. Now, as $n_{3} \rightarrow 0$, the point $\zeta_{3}$ goes to infinity and, hence, the line $\left(z_{1}, z_{2}\right)$ becomes an asymptote of the hyperbola.

3. Sections of a linear partial fraction. In order to generalize Theorem 1 , let us now assume that $n=m_{1}+m_{2}+\cdots+m_{p} \neq 0, n_{1}=m_{1}$ $+m_{2}+\cdots+m_{k} \neq 0, n_{2}=n-n_{1} \neq 0$.

Let us write

$$
\begin{aligned}
& F(z)=\sum_{j=1}^{p} \frac{m_{j}}{z-z_{j}}=\frac{n\left(z-Z_{1}\right)\left(z-Z_{2}\right) \cdots\left(z-Z_{p-1}\right)}{\left(z-z_{1}\right)\left(z-z_{2}\right) \cdots\left(z-z_{p}\right)}, \\
& F_{1}(z)=\sum_{j=1}^{k} \frac{m_{j}}{z-z_{j}}=\frac{n_{1}\left(z-z_{1}^{\prime}\right)\left(z-z_{2}^{\prime}\right) \cdots\left(z-z_{k-1}^{\prime}\right)}{\left(z-z_{1}\right)\left(z-z_{2}\right) \cdots\left(z-z_{k}\right)}, \\
& F_{2}(z)=\sum_{j=k+1}^{p} \frac{m_{j}}{z-z_{j}}=\frac{n_{2}\left(z-z_{1}^{\prime \prime}\right)\left(z-z_{2}^{\prime \prime}\right) \cdots\left(z-z_{p-k-1}^{\prime \prime}\right)}{\left(z-z_{k+1}\right)\left(z-z_{k+2}\right) \cdots\left(z-z_{p}\right)} .
\end{aligned}
$$


Since $F(z)=F_{1}(z)+F_{2}(z)$, it follows that

$$
\begin{aligned}
n\left(z-Z_{1}\right) \cdots(z- & \left.Z_{p-1}\right) \\
= & n_{1}\left(z-z_{1}^{\prime}\right) \cdots\left(z-z_{k-1}^{\prime}\right)\left(z-z_{k+1}\right) \cdots\left(z-z_{p}\right) \\
& \quad+n_{2}\left(z-z_{1}\right) \cdots\left(z-z_{k}\right)\left(z-z_{1}^{\prime \prime}\right) \cdots\left(z-z_{p-k-1}^{\prime \prime}\right) .
\end{aligned}
$$

Hence, if $z^{\prime \prime}$ is any $z_{j}^{\prime \prime}$ or a point $z_{j}$ with $1 \leqq j \leqq k$, and if $Z$ is any point $Z_{j}$, we find that

$$
\begin{gathered}
\frac{\left(z^{\prime \prime}-Z_{1}\right) \cdots\left(z^{\prime \prime}-Z_{k-1}\right)\left(z^{\prime \prime}-Z_{k}\right) \cdots\left(z^{\prime \prime}-Z_{p-1}\right)}{\left(z^{\prime \prime}-z_{1}^{\prime}\right) \cdots\left(z^{\prime \prime}-z_{k-1}^{\prime}\right)\left(z^{\prime \prime}-z_{k+1}\right) \cdots\left(z^{\prime \prime}-z_{p}\right)}=\frac{n_{1}}{n}, \\
\frac{\left(Z-z_{1}^{\prime}\right) \cdots\left(Z-z_{k-1}^{\prime}\right)\left(Z-z_{k+1}\right)\left(Z-z_{k+2}\right) \cdots\left(Z-z_{p}\right)}{\left(Z-z_{1}\right) \cdots\left(Z-z_{k-1}\right)\left(Z-z_{k}\right)\left(Z-z_{1}^{\prime \prime}\right) \cdots\left(Z-z_{p-k-1}^{\prime \prime}\right)}=-\frac{n_{2}}{n_{1}} .
\end{gathered}
$$

Now, taking the moduli and amplitudes of equations (3.1) and (3.2), we derive the following theorem.

TheOREM 3. Given the partial fraction $F(z)=\sum_{1}^{p} m_{j}\left(z-z_{j}\right)^{-1}$ and its sections $F_{1}(z)=\sum_{1}^{k} m_{j}\left(z-z_{j}\right)^{-1}$ and $F_{2}(z)=\sum_{k+1}^{p} m_{j}\left(z-z_{j}\right)^{-1}$, where $n_{1}=\sum_{1}^{k} m_{j} \neq 0, n_{2}=\sum_{k+1}^{p} m_{j} \neq 0$, and $n=n_{1}+n_{2} \neq 0$. Denote by $S$ the set of $p-1$ zeros of $F(z)$; by $S_{1}$ the set of $p-1$ points comprised of the $k-1$ zeros of $F_{1}(z)$ and the $p-k$ points $z_{k+1}, z_{k+2}, \cdots, z_{p}$; and, by $S_{2}$, the set of $p-1$ points comprised of the $k$ points $z_{1}, z_{2}, \cdots, z_{k}$ and the $p-k$ -1 zeros of $F_{2}(z)$.

A. Then, if $z^{\prime \prime}$ is any point of $S_{2}$, the product of its distances to the points of $S$ is $\left|n_{1} / n\right|$ times the product of its distances to the points of $S_{1}$. Also, if each point in $S$ is paired in any manner with a point in $S_{1}$, the sum of the angles subtended at $z^{\prime \prime}$ by the $p-1$ pairs of points is zero or $\pi(\bmod 2 \pi)$ according as $n_{1} n>0$ or $<0$.

B. Further, if $Z$ is any point of $S$, the product of its distances to the points of $S_{1}$ is $\left|n_{2} / n_{1}\right|$ times the product of its distances to the points in $S_{2}$. Also, if each point in $S_{1}$ is paired in any manner with a point in $S_{2}$, the sum of the angles subtended at $Z$ by the $p-1$ pairs of points is zero or $\pi(\bmod 2 \pi)$ according as $n_{1} n_{2}<0$ or $>0 .^{3}$

For example, if $p=4$ and $k=2$, then $F_{1}(z)$ has as its only zero the point $z^{\prime}$ dividing the line segment $\left(z_{1}, z_{2}\right)$ in the ratio $m_{1}: m_{2}$ and $F_{2}(z)$ has as its only zero the point $z^{\prime \prime}$ dividing the line segment $\left(z_{3}, z_{4}\right)$ in

${ }^{3}$ In the case $m_{1}=m_{2}=\cdots=m_{p}=k=1$, the set $S_{2}$ consists of the point $z_{1}$ and the $p-2$ zeros of $F_{2}(z)$. Bôcher (see footnote 1 ) states without proof Theorem 3 , but takes the point $z^{\prime \prime}$ to be only the point $z_{1}$, making no mention of the fact that $z^{\prime \prime}$ may be also any of the other $p-2$ points of $S_{2}$. In Theorem 3, the sense in which an angle subtended by a line segment is to be measured may be determined from formulas (3.1) and (3.2). 
the ratio $m_{3}: m_{4}$. Using part $\mathrm{B}$ of Theorem 3 , we may prove that the sum of the angles subtended in any zero $Z$ of $F(z)$ by the line segments $\left(z^{\prime}, z_{1}\right)$ and $\left(z_{4}, z^{\prime \prime}\right)$ is the supplement of the angle subtended in $Z$ by the segment $\left(z_{2}, z_{3}\right)$. Likewise, by using part $\mathrm{A}$, we may prove that, if $\psi$ denotes double the angle from the line $\left(z_{3}, z_{4}\right)$ to the line $\left(z_{1}, z_{2}\right)$, then $(\psi-\pi)$ equals the sum of the angles subtended by the line segment $\left(z^{\prime}, z^{\prime \prime}\right)$ in the zeros $Z_{1}, Z_{2}$, and $Z_{3}$ of $F(z)(\bmod 2 \pi)$.

4. Geometric interpretations of Theorem 3. First, Theorem 3 may be restated in terms of two types of generalizations of the circle which were studied by Darboux.. ${ }^{4}$ One is the so-called lemniscate, the locus of a point which moves so that the product of its distances from one set of fixed points (called poles) is a constant multiple of the product of its distances from another set of fixed points (also called poles). The other is the so-called stelloid, the locus of a point which moves so that the sum of the angles subtended in it by a set of pairs of fixed points (called poles) is constant. These two families are respectively the equipotential curves and lines of force in the field due to unit attractive particles at the points of the one set and unit repulsive particles at the points of the other set. Thus, according to Theorem 3, the points of $S_{2}$ lie at the intersections of a certain lemniscate and a certain stelloid, both of which have the points of $S$ and $S_{1}$ as poles, whereas the points of $S$ lie at the intersections of a lemniscate and a stelloid, both of which have the points of $S_{1}$ and $S_{2}$ as poles.

Secondly, Theorem 3 may be used in combination with Theorem 2. By Theorem 2, the points $Z_{1}, Z_{2}, \cdots, Z_{p-1}$ are the foci of the curve $C_{0} \equiv C\left(z_{1}, \cdots, z_{p} ; m_{1}, \cdots, m_{p}\right)$ of class $p-1$; the points $z_{1}^{\prime}, z_{2}^{\prime}, \cdots, z_{k-1}^{\prime}$ the foci of the curve $C_{1} \equiv C\left(z_{1}, \cdots, z_{k} ; m_{1}, \cdots, m_{k}\right)$ of class $k-1$; and the points $z_{1}^{\prime \prime}, z_{2}^{\prime \prime}, \cdots, z_{p-k-1}^{\prime \prime}$ the foci of the curve $C_{2} \equiv C\left(z_{k+1}, \cdots, z_{p} ; m_{k+1}, \cdots, m_{p}\right)$ of class $p-k-1$. Thus, if $p=6$ and $k=3$ and all $m_{j}>0$, the points $z_{1}^{\prime}$ and $z_{2}^{\prime}$ are the foci of the ellipse which touches the line segments $\left(z_{1}, z_{2}\right),\left(z_{2}, z_{3}\right)$ and $\left(z_{3}, z_{1}\right)$ in points dividing these segments in the respective ratios $m_{1}: m_{2}, m_{2}: m_{3}$ and $m_{3}: m_{1}$, while the points $z_{1}^{\prime \prime}$ and $z_{2}^{\prime \prime}$ are the foci of the ellipse which touches the line segments $\left(z_{4}, z_{5}\right),\left(z_{5}, z_{6}\right)$ and $\left(z_{6}, z_{4}\right)$ in the points dividing these segments in the respective ratios $m_{4}: m_{5}, m_{5}: m_{6}$ and $m_{6}: m_{4}$.

The set $S$ of Theorem 3 consists thus of the foci of the curve $C_{0}$. The set $S_{1}$ consists thus of the points $z_{k+1}, z_{k+2}, \cdots, z_{p}$ and the foci of the curve $C_{1}$ or, what amounts to the same, the foci of the degen-

${ }^{4} \mathrm{G}$. Darboux, Sur une classe remarquable de courbes et de surfaces algebrique, Paris, 1873, pp. 66-73. 
erate curve $C^{\prime} \equiv C\left(z_{1}, \cdots, z_{k}, z_{k+1}, \cdots, z_{p} ; m_{1}, \cdots, m_{k}, 0, \cdots, 0\right)$ of class $p-1$. Similarly the set $S_{2}$ may be regarded as consisting of the foci of the degenerate curve $C^{\prime \prime}=C\left(z_{1}, \cdots, z_{k}, z_{k+1}, \cdots, z_{p}\right.$; $\left.0, \cdots, 0, m_{k+1}, \cdots, m_{p}\right)$, also of class $p-1$. Thus, Theorem 3 expresses two geometric relations regarding the foci of the three curves $C_{0}, C^{\prime}$ and $C^{\prime \prime}$ of class $p-1$.

5. Further generalizations. It is obvious that Theorem 3 may be extended at once to the more general partial fractions

$$
F(z)=\sum_{j=1}^{p} \frac{m_{j}\left(z-a_{j 1}\right) \cdots\left(z-a_{j r}\right)}{\left(z-b_{j 1}\right) \cdots\left(z-b_{j s}\right)} .
$$

For example, let us take all $m_{j}>0$, and

$$
F(z)=\sum_{j=1}^{3} \frac{m_{j}}{\left(z-z_{j}\right)^{q}}, \quad F_{1}(z)=\frac{m_{1}}{\left(z-z_{1}\right)^{q}}, \quad F_{2}(z)=F(z)-F_{1}(z) .
$$

The zeros of $F_{2}(z)$ are the roots of the equations

$$
z-z_{2} / z-z_{1}=\left(m_{2} / m_{1}\right)^{1 / q} \exp [\pi(2 k+1) i / q],
$$

where $k=0,1,2, \cdots, q-1$. These zeros may therefore be located as the intersections of the circle $\left|z-z_{2}\right|=\left(m_{2} / m_{1}\right)^{1 / q}\left|z-z_{1}\right|$ with the $q$ arcs of circles arg $\left(z-z_{2}\right) /\left(z-z_{1}\right)=\pi(2 k+1) / q, k=0,1,2, \cdots, q-1$. On the other hand, if $\zeta$ is any zero of $F_{2}(z)$,

$$
\frac{\left(\zeta-z_{1}\right)^{q}}{n} F(\zeta)=\frac{m_{1}}{n}=\frac{\left(\zeta-Z_{1}\right) \cdots\left(\zeta-Z_{2 q}\right)}{\left(\zeta-z_{2}\right)^{q}\left(\zeta-z_{3}\right)^{q}},
$$

where $Z_{1}, Z_{2}, \cdots, Z_{2 q}$ are the zeros of $F(z)$ and $n=m_{1}+m_{2}+m_{3}$. From equation (5.1) we may thus conclude that the sum of the angles subtended in $\zeta$ by the segments from $z_{2}$ to any $q$ of the points $Z_{j}$ equals (modulo $2 \pi$ ) the sum of the angles subtended in $\zeta$ by the segments from the remaining $Z_{j}$ to $z_{3}$.

University OF Wisconsin in MiLwaukee 